\title{
Barriers Experienced by Medical Students in Conducting Research at Undergraduate Level
}

Jai Kumar, Aurangzeb Memon, Ankeet Kumar, Raj Kumari, Besham Kumar, Sundus Fareed 1.

Corresponding author: Sundus Fareed, sundus_fareed@yahoo.com

\section{Abstract \\ Introduction}

Undergraduate medical research is very important not only for scientific learning but also for career progress. However, there are barriers, especially in developing countries, that restrict undergraduate research. This study aims to evaluate the barriers experienced by medical students in conducting research at undergraduate level.

\section{Methods}

It was an observational, cross-sectional survey conducted with 687 clinical students of two public medical universities of Pakistan. A self-structured questionnaire consisting of seven items was administered to assess the barriers in conducting research at undergraduate level. Data was processed and analysed through SPSS v 22.0 (IBM Corp., Armonk, NY, USA).

\section{Results}

Lack of knowledge as a barrier was identified by $90.68 \%(n=623)$ students. The second most common barrier identified by the students was lack of time $(88.79 \% ; n=610)$, followed by lack of mentoring as the third most common barrier $(85.74 \%$; $n=572)$. Sub-group analysis showed that lack of knowledge, lack of mentoring, limited data base access, lack of time, and lack of finances were more crucial barriers for female gender $(\mathrm{p}<0.05)$. Only lack of interest was a crucial barrier for male gender $(\mathrm{p}<0.05)$.

\section{Conclusion}

A number of barriers need to be addressed in order to enhance students' participation in clinical research such as lack of interest, funding, and poor availability of research mentors and access to scientific databases to improve participation in clinical research. Substantial

Received 04/02/2019

Review began 04/04/2019 Review ended 04/07/2019 Published 04/13/2019

\section{(c) Copyright 2019}

Kumar et al. This is an open access article distributed under the terms of the Creative Commons Attribution License CC-BY 3.0., which permits unrestricted use, distribution, and reproduction in any medium, provided the original author and source are credited.
Categories: Medical Education, Miscellaneous, Other

Keywords: medical students, clinical research, barriers, pakistan

\section{Introduction}

Modern medicine and healthcare depend and evolve on the basis of evidences which lead to better understanding of the diseases. It is the main reason why physicians should have keen interest in medical research [1]. Considering the importance of research in understanding 
diseases in all aspects, undergraduate clinical research is currently more valuable and more desperately needed than before [2]. Involving medical students in clinical research activities at undergraduate level boosts their interest and improves their scientific output as opposed to their peers who are not involved in research. Students who have published articles as undergraduate publish three times more articles later as doctors as compared to those students who were not involved in research at student level [3]. A systematic review by Straus et al. stated that having a medical school publication positively influences academic career choice among medical students [4].

Benjamin in his study stated that students in developing and low-income countries face more difficulties in conducting research than developed countries [5]. Only $1.2 \%$ of researches globally come from South Asia, and the situation is ever graver in Pakistan due to lack of physician-scientists [6]. Lack of research training in medical students has been held as the primary reason behind unavailability of undergraduate research. Other barriers can be both professional and personal barriers. Professional barriers may include limited access to information, limited access to equipment, and lack of mentorship. Personal barriers may include inadequate knowledge of research methodology, inadequate statistic skills, and time and financial constraint $[7,8]$.

There is a lack of data available for barriers among medical students in Pakistan in conducting research. In order to allow our medical students flourish academically and scientifically in comparison to peers from developed country, it is important to identify these barriers.

\section{Materials And Methods}

\section{Study design, settings, and participants}

It was an observational, cross-sectional survey conducted in two public medical universities of Pakistan in February 2019. Nine hundred and twelve clinical students (3rd-5th year) were invited to participate, 707 students agreed to participate (response rate: $77.5 \%$ ). Twenty responses were excluded due to incomplete information. Hence, there were 687 students who completed the survey.

\section{Study instrument}

A self-administered questionnaire was structured. As a pilot project, 50 students of final year were requested to mention any five barriers that they have faced in conducting research. Total of nine items were identified, of which two were removed because of redundancy and the final questionnaire comprised of seven items. These included lack of mentoring, time constraint, lack of research knowledge, lack of interest, lack of research culture, limited access to database and lack of finances. Participants of the actual survey were requested to randomly select any or all barriers that were relevant to them.

\section{Data analysis}

Data was processed and analyzed using SPSS for Windows version 22.0 (IBM Corp., Armonk, NY, USA). Internal consistency of the seven items was calculated using Cronbach alpha. Frequency and percentage for each item was calculated. Sub-analysis was done to assess the correlation of gender on these barriers using chi-square. $\mathrm{P}$ value $\leqslant 0.05$ was considered significant.

\section{Results}

The study included 687 participants, of these 521 (75.83\%) were females and 166 (24.17\%) were males. 


\section{Cureus}

The internal consistency of the seven-item scale was 0.78 in this study. Lack of knowledge as a barrier was identified by $90.68 \%$ (623 out of 687) students. The second most common barrier identified by the students was lack of time (88.79\%; 610 out of 687$)$, followed by lack of mentoring as the third most common barrier (85.74\%; 572 out of 687). The common barriers experienced by medical students in conducting research are shown in Figure 1.

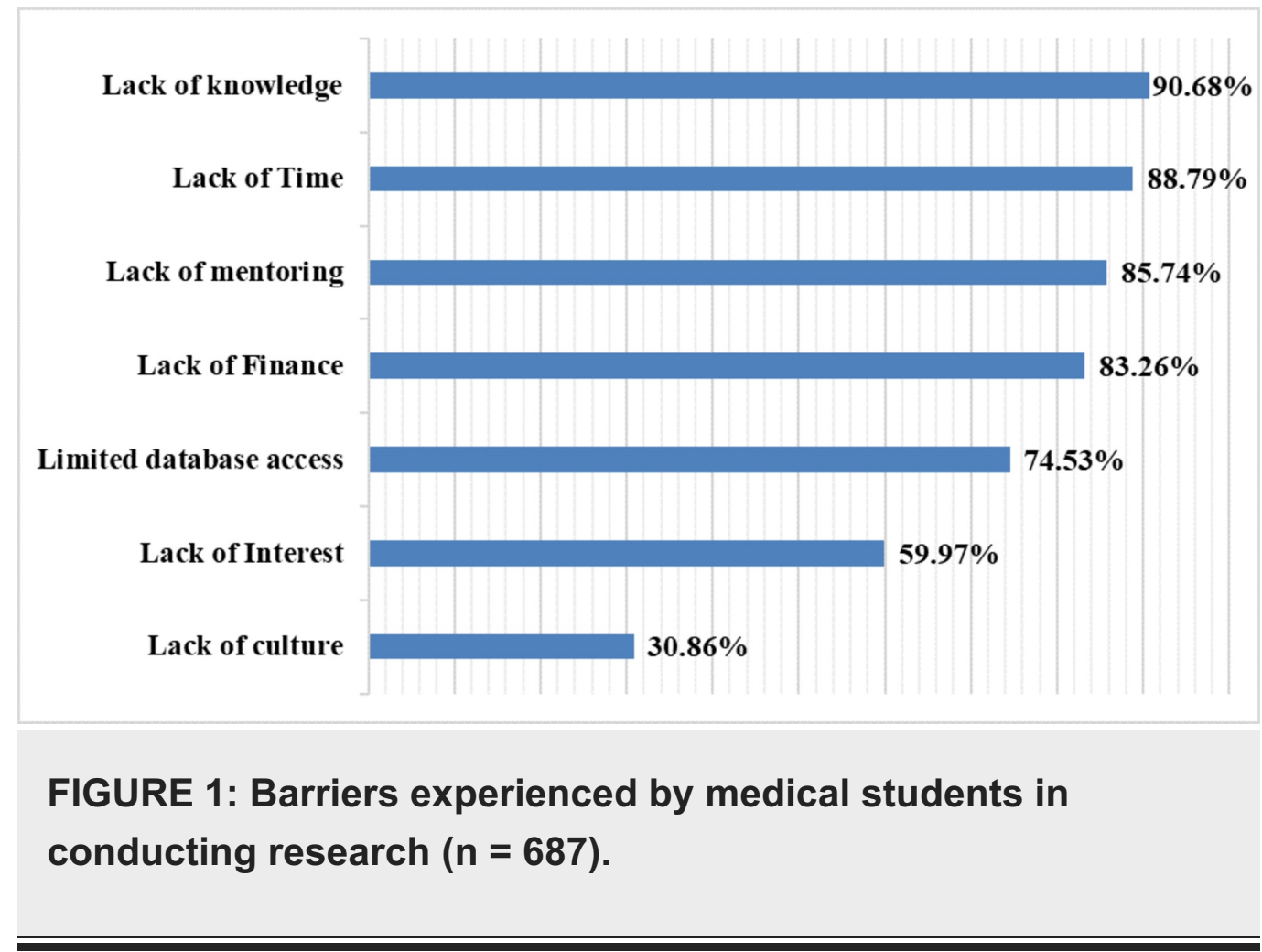

Sub-group analysis showed that lack of knowledge, lack of mentoring, limited data base access, lack of time, and lack of finances were more crucial barriers for female gender. Only lack of interest was a crucial barrier for male gender $(P$ value $=0.0001)$. There was no significant difference in lack of culture barrier as shown in Table 1. 


\section{Cureus}

\begin{tabular}{|c|c|c|c|}
\hline Barriers & Male n (\%) & Female n (\%) & $P$ value \\
\hline Lack of knowledge & $122(73.49 \%)$ & 501 (96.16\%) & 0.0001 \\
\hline Limited database access & $95(57.23 \%)$ & 417 (80.04\%) & 0.0001 \\
\hline Lack of culture & $41(24.70 \%)$ & $171(32.82 \%)$ & 0.05 \\
\hline Lack of mentoring & $87(52.41 \%)$ & 502 (96.35\%) & 0.0001 \\
\hline Lack of interest & $128(77.11 \%)$ & $284(54.51 \%)$ & 0.0001 \\
\hline Lack of time & $113(68.07 \%)$ & $497(95.39 \%$ & 0.0001 \\
\hline Lack of finance & $129(77.71 \%)$ & $443(85.03 \%)$ & 0.03 \\
\hline
\end{tabular}

TABLE 1: Impact of gender on the barriers experienced in conducting research $(n=$ 687).

\section{Discussion}

Although, the importance of research is well known and repeatedly stated in the field of medicine, only a small proportion of medical students conduct research $[9,10]$. There are many barriers that are responsible for this deficit. In this study, it was seen that major barriers in conducting clinical research as medical students include lack of knowledge, lack of finance, lack of time and lack of mentorship. These results were consistent with the outcomes another study conducted with Egyptian medical school in 2016 [7].

In this study, 90.68\% participants stated lack of knowledge regarding research methodology and other aspect of research as their core reason for not conducting clinical research. Bennett et al. also had similar findings regarding lack of knowledge as challenge for research [11]. It is shown that mandatory participation in research activity improves student knowledge [12].

In this study, 88.79\% participants stated lack of time as their reason for not conducting clinical research. Lack of time was the major stated reason in a study conducted in Egypt [7]. Similar findings were also stated in Saudi students where $72 \%$ of the students consider lack of time as the barrier to conduct research [9]. Siemens et al., in 2010, considered lack of time as a significant barrier in conducting research due to busy academic schedule [10].

In this study, $85.74 \%$ of the participants considered lack of mentorship is the main factor stopping them from conducting research. Acquiring new skills such as conducting research is easy when one has a mentor that guides through the learning phase. Mentors not only facilitate in learning skills but also assess progress and advise corrective measures [13]. The results were echoed by similar studies regarding importance of research mentorship for medical students [7, 10].

In this study, lack of finances or funding was considered a barrier by $83.26 \%$ participants. Hegde et al. mentioned lack of funding from institutions as the chief barrier for undergraduate research [14].

In this study, limited access to database was also reported as a barrier by more than $59 \%$ of the 
participants. This finding was also consistent in Egyptian medical students [7]. Silva et al. in his study stated that, compared to peers in developed countries, medical students have limited access to database in low income and developing countries [15].

Based on this study, we suggest the need of substantial amendments in the medical undergraduate curriculum. Lack of knowledge was the most common barrier, hence, we recommend interactive sessions and workshops on all aspects of medical research from early undergraduate years. Lack of time was found as a significant barrier against research; therefore, we suggest making research participation mandatory for the students. Research methodology and data analysis should be taught in the classes and it should be included in their final grading. Faculty should mentor the students and encourage them to participate in research activities. In-campus digital access to various databases and online research resources must be provided to the students to boost their interest and streamline the process of conducting medical research for them.

\section{Conclusions}

The major barriers that were experienced by medical students in conducting research in this survey were lack of knowledge, lack of time, lack of mentoring and lack of finance. These hindrances need to be addressed in order to enhance students' participation in clinical research.

\section{Additional Information Disclosures}

Human subjects: Consent was obtained by all participants in this study. Advance Educational Institute and Research Center issued approval MU/ECA/19/022. Animal subjects: All authors have confirmed that this study did not involve animal subjects or tissue. Conflicts of interest: In compliance with the ICMJE uniform disclosure form, all authors declare the following: Payment/services info: All authors have declared that no financial support was received from any organization for the submitted work. Financial relationships: All authors have declared that they have no financial relationships at present or within the previous three years with any organizations that might have an interest in the submitted work. Other relationships: All authors have declared that there are no other relationships or activities that could appear to have influenced the submitted work.

\section{References}

1. Murdoch-Eaton D, Drewery S, Elton S, et al.: What do medical students understand by research and research skills? Identifying research opportunities within undergraduate projects. Med Teach. 2010, 32:152-160. 10.3109/01421591003657493

2. Wyngaarden JB: The clinical investigator as an endangered species . N Engl J Med. 1979, 301:1254-1259. 10.1056/NEJM197912063012303

3. Reinders JJ, Kropmans TJB, Cohen-Schotanus J: Extracurricular research experience of medical students and their scientific output after graduation. Med Edu. 2005, 39:237. 10.1111/j.1365-2929.2004.02078.x

4. Straus SE, Straus C, Tzanetos K: Career choice in academic medicine: systematic review . J Gen Int Med. 2006, 21:1222-1229. 10.1111/j.1525-1497.2006.00599.x

5. Puertas EB, Arósquipa C, Gutiérrez D: Factors that influence a career choice in primary care among medical students from high-, middle-, and low-income countries: a systematic review. Rev Panam Salud Publica. 2013, 34:351-358.

6. Solomon SS, Tom SC, Pichert J, Wasserman D, Powers AC: Impact of medical student research in the development of physician-scientists. J Investig Med. 2003, 51:149-156. 10.1136/jim-5103-17

7. Abushouk AI, Hatata AN, Omran IM, Youniss MM, Elmansy KF, Meawad AG: Attitudes and 
perceived barriers among medical students towards clinical research: a cross-sectional study in an Egyptian medical school. J Biomed Edu. 2016, 2016:7. 10.1155/2016/5490575

8. Dadipoor S, Ramezankhani A, Aghamolaei T, Safari-Moradabadi A: Barriers to research activities as perceived by medical university students: a cross-sectional study. Avicenna J Med. 2019, 9:8-14. 10.4103/ajm.AJM_121_18

9. Alghamdi KM, Moussa NA, Alessa DS, Alothimeen N, Al-Saud AS: Perceptions, attitudes and practices toward research among senior medical students. Saudi Pharm J. 2014, 22:113-117. 10.1016/j.jsps.2013.02.006

10. Siemens DR, Punnen S, Wong J, Kanji N: A survey on the attitudes towards research in medical school. BMC Med Educ. 2010, 10:4. 10.1186/1472-6920-10-4

11. Bennett NR, Cumberbatch C, Francis DK: There are challenges in conducting systematic reviews in developing countries: the Jamaican experience. J Clin Epidemiol. 2015, 68:10951098. 10.1016/j.jclinepi.2014.09.026

12. Segal S, Lloyd T, Houts PS, Stillman PL, Jungas RL, Greer RB: The association between students' research involvement in medical school and their postgraduate medical activities. Acad Med. 1990, 65:530-533. 10.1097/00001888-199008000-00010

13. Ward EC, Hargrave C, Brown E, Halkett G, Hogg P: Achieving success in clinically based research: the importance of mentoring. J Med Radiat Sci. 2017, 64:315-320. 10.1002/jmrs.234

14. Hegde A, Venkataramana G, Kulkarni SB, Bhaskar NN, Jacob J, Gangadharappa SK: Attitudes, experiences, and barriers to research and publishing among dental postgraduate students of Bengaluru City: a cross-sectional study. J Ind Assoc Public Health Dent. 2017, 15:157-161. 10.4103/jiaphd.jiaphd_11_17

15. Silva TD, da Cunha Aguiar LC, Leta J, et al.: Role of the undergraduate student research assistant in the new millennium. Cell Biol Educ. 2004, 3:235-240. 10.1187/cbe.04-02-0032 